\section{LOW VOLTAGE SCANNING ELECTRON MICROSCOPY AND JACK RAMSEY'S PRINCIPLE}

\author{
Oliver C. Wells, \\ IBM Research Division \\ wellsoc@research.ibm.com
}

"Low Voltage Scanning Electron Microscopy (LVSEM), defined as operation in the energy range below $5 \mathrm{keV}^{\prime \prime}$ was described by David Joy and Dale Newbury (1) in the previous issue of this journal as being "perhaps the most important single operational mode of the SEM." In their paper they describe both the advantages and disadvantages of this method.

Ramsey's principle, which was established by Jack Ramsey over many years as a microscopist and microanalyst, is to say: "There is no best way of doing ANYTHING."

The purpose of this note is to give some examples in which Ramsey's principle was applied to the choice of the beam energy in the SEM, the point being that this can reveal unexpected information in some cases and in a way that might not be obvious in advance of the experiment.

First example: An example from routine service work is shown in Fig.1. Here, an oxidized silicon wafer had been covered with $30 \mathrm{~nm}$ of $\mathrm{Cr}, 150 \mathrm{~nm}$ of Si and $150 \mathrm{~nm}$ of Au. This was heat-treated for 1 hour in $\mathrm{He}$ at $300^{\circ} \mathrm{C}$. to give a contact area. The microscopist (the late Phil Bailey) was asked to find

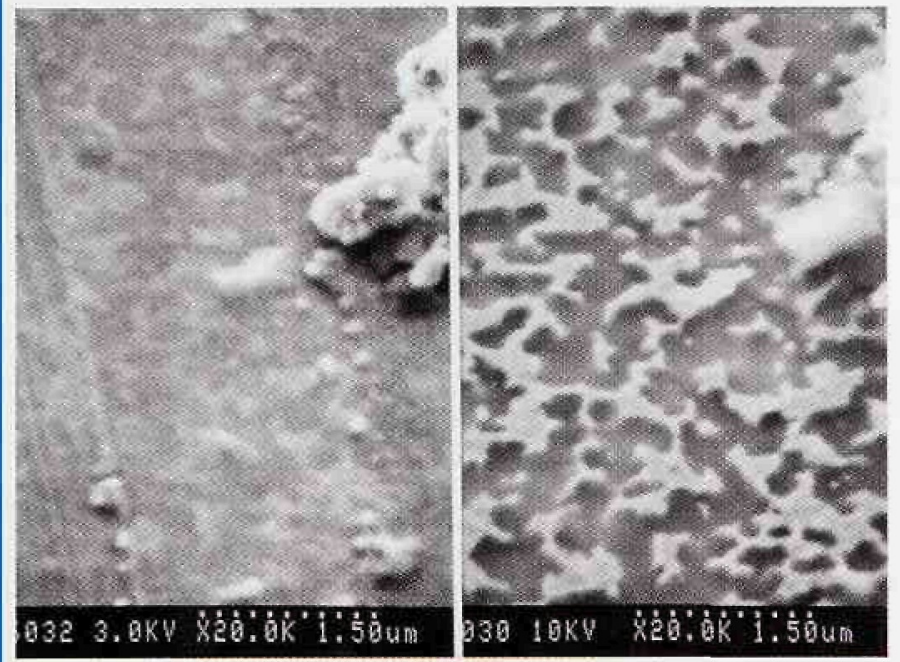

Figure 1. SE images of sample with sub-surface structures with beam energies: (a) $3 \mathrm{keV}$ and (b) $10 \mathrm{keV}$. Field of view measures 3.75 micrometers left to right. (Micrographs taken by P.J. Bailey.)

out whether the metal layers had mixed together uniformly or if there were regions of different composition.(2)

Fig. 1(b) on the right shows his first image, obtained with a beam energy of $10 \mathrm{keV}$. This seems to show that the films have segregated to give alternating regions of two different compositions. And yet, this secondary electron (SE) image looks "wrong" having the appearance of compositional contrast in the backscattered electron (BSE) image. So he reduced the beam energy to $3 \mathrm{keV}$ and obtained Fig.1(a) which shows the surface topography in the manner of the LVSEM, and where these compositional contrasts have disappeared.

From this comparison pair of micrographs it is clear that the segregated regions are in a layer below the surface, a fact that was verified soon enough by breaking the sample and looking at the cross-section. (At first sight the sharpness of such an image of the underlying structure may come as a surprise, and arises because a certain fraction of the beam re- mains compact - except for "beam broadening" - during „u " „tial stages of penetration into the specmen(3).)

Second example: An example that was obtained and used in lectures by the late Harry Carter is shown in Fig.2. It shows a comparison pair of SE images of a louse egg with an open lid. The SE image obtained at $10 \mathrm{keV}$ on the left shows the surface topography without showing any of the underlying struc-
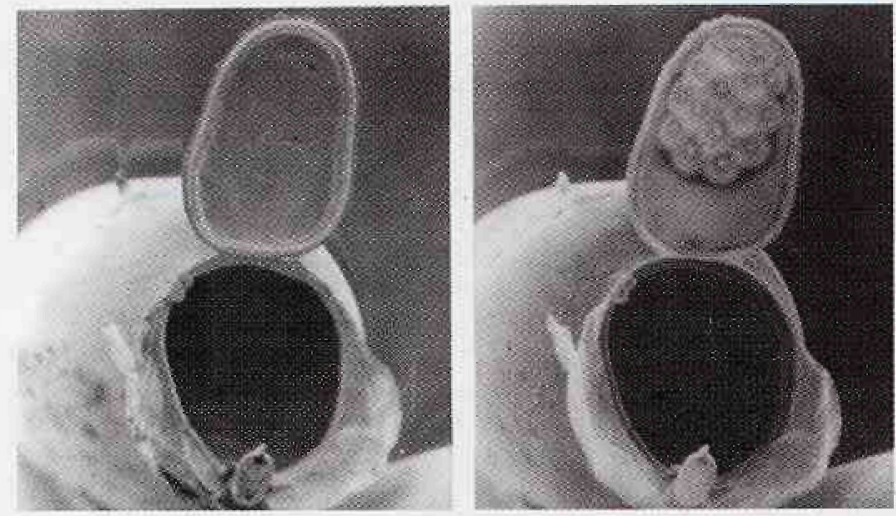

Figure 2. SE images of louse egg (pthirus pubis Linnaeus)with beam energies: (a) $10 \mathrm{keV}$ and (b) $25 \mathrm{keV}$. Field of view measures $1 \mathrm{~mm}$ left to right. (Micrographs by H.W. Carter.)

ture. At $25 \mathrm{keV}$ the beam can penetrate into the lid to show the underlying breathing cavity. The effects of penetration can also be seen at $25 \mathrm{keV}$ at the membrane that surrounds the opening into the egg.

In general, to show the surface of a biological sample, the beam energy must be low so as to give a good spatial resolution(4), while to show cavities or high- $Z$ regions below the surface it must be increased to typically several tens of keV.

The above two examples show how extra valuable information can be obtained by comparing the images at two different beam energies. In neither case was it obvious in advance that this would be the case, and this shows the diligence of the microscopist in each case.

\section{University of Massachusetts Amherst Professional Microscopy Position}

Microscopist sought to participate in research projects and assist and train clients in the Central Microscopy Facility ( www.bio.umass.edu/ microscopy ). Opportunity to learn new skills and advanced techniques. MS in a Biological Science area and at least 3 years of experience in transmission electron microscopy is required for this benefited full-time position. Competitive salary commensurate with experience. Send CV with three names and e-mail addresses of references to Microscopy Search, clo Dr. Chris Woodcock, Biology Department, University of Massachusetts, Amherst, MA 01003 (chris@bio.umass.edu). Evaluation of applicants will begin June 1st 2002 and continue until the position is filled. The University of Massachusetts is an Equal Opportunity Affirmative Action employer. 


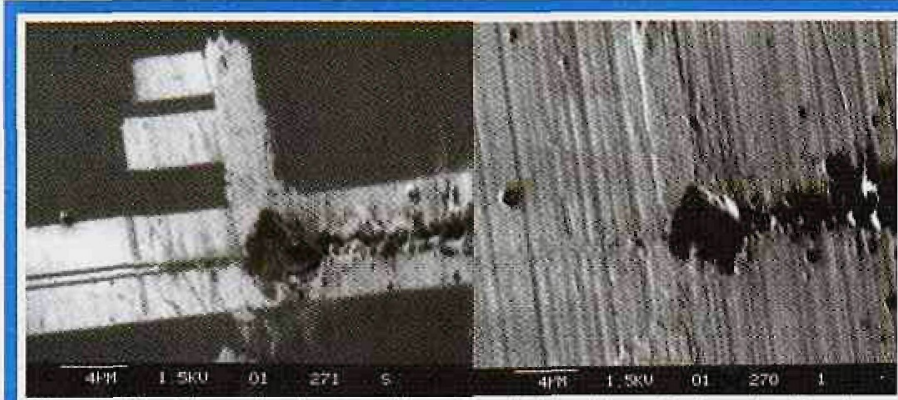

Figure 3 . Uncoated recording head tilted by $45 \mathrm{deg}$. to the lowloss electron (LLE) detector on the left.(5) Beam energy $=1.5$ keV to minimise charging: (a) SE image. (b) LLE image from the same field of view. Energy window $=500 \mathrm{eV}$. Field of view measures 30 micrometers left to right.

Third example: An example in which an unexpected advantage was obtained by the use of two different electron detector systems is shown in Fig.3. The sample was a thin-film magnetic recording head, in which metal polepieces were embedded in a matrix of non-conducting material (alumina).(5) In this case to avoid charging the beam energy must be set equal to the "second crossover energy" where the sum of the SE and BSE coefficients is equal to unity.(6) In the example shown, this seemed to be at about $1 \mathrm{keV}$, with violent charging either above or below this value. (In such a case it can be very helpful to integrate the image using typically 16 or 32.4 -second scans with an integrating image store.)

The unexpected feature of this sample was an overlying thin layer of lubricant that could not be seen in the SE image
(Fig.3a). The low-loss electron image is obtained from a surface layer of thickness typically measured in tens of $\mathrm{nm}$ and shows this fact clearly (Fig.3b). The above examples were given to emphasise once again the practical significance of Ramsey's principle according to which you must ALWAYS vary SOMETHING and then keep your eyes open for whatever totally unexpected - but usually valuable - extra piece of information will be revealed. (And you must never take the sample out of the microscope too quickly in case you might then think of something else to change.)

The images shown here changed significantly when the chosen parameter was changed. In the majority of cases the changes may be considerably less dramatic but can be very useful even in such less imposing cases.

(1) Joy, D.C., Newbury, D.E., (2002), 'Low Voltage Scanning Electron Microscopy ${ }^{\prime}$ Microscopy Today March/April 2002, p. 22 only.

(2) Wells, O.C., Bailey, P.J. (1986), 'Imaging of sub-surface structures in a solid specimen in the SEM' EMSA Bulletin 16, 76-77.

(3) Gentsch, P., Reimer. L., (1973), Measurements of the Resolution in the Scanning Transmission Electron Microscopy of Thick Objects' (in German with English abstract), Optik 37, 451-454.

(4) Ardenne, M.von, (1940), 'Elektronen-Uebermikroskopie', Springer, Berlin (1940); Edwards, Ann Arbor (1943).

(5) Wells, O.C., (2002), "Imaging of silicon integrated circuits by the lowloss electron (LLE) method in the scanning electron microscope (SEM)', Proc. ALC'01, Nara, Japan, in press.

(6) Knoll, M., (1935), 'Static potential and secondary emission of bodies under electron irradiation' (in German), Z. Tech.Phys. 11, 467-475.

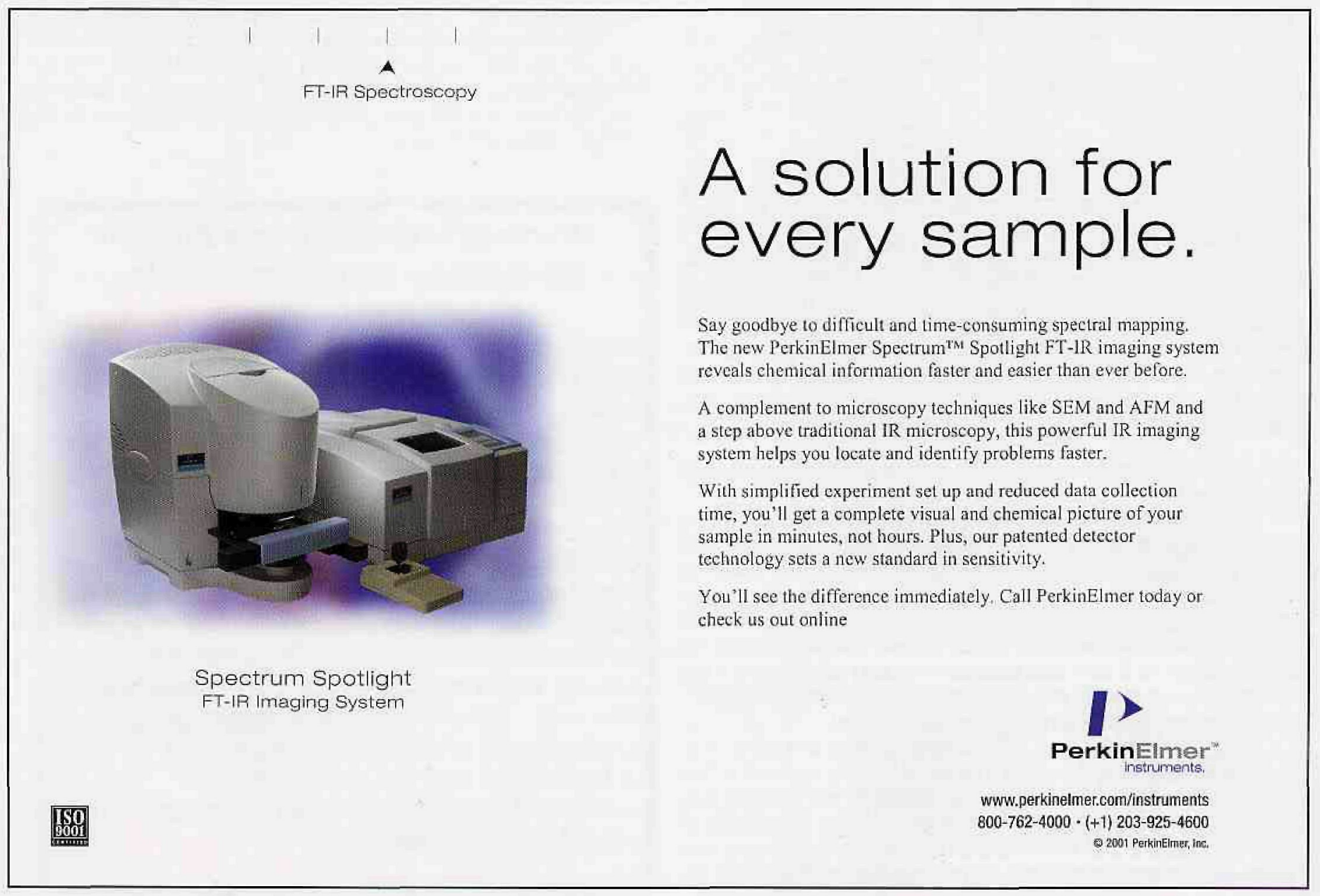

